\title{
The developmental trajectory of children's auditory and visual statistical learning abilities: modality-based differences in the effect of age
}

\author{
Limor Raviv ${ }^{1,3}$ | Inbal Arnon ${ }^{2}$
}

${ }^{1}$ Psychology of Language Department, Max Planck Institute for Psycholinguistics,

Nijmegen, The Netherlands

${ }^{2}$ Department of Psychology, Hebrew

University, Jerusalem, Israel

${ }^{3}$ Department of Cognitive Science, Hebrew University, Jerusalem, Israel

\section{Correspondence}

Limor Raviv, Max Planck Institute for

Psycholinguistics, Wundtlaan 1, 6525 XD

Nijmegen, The Netherlands.

Email: limor.raviv@mpi.nl

Funding information

Israel Science Foundation, Grant/Award

Number: 584/16

\begin{abstract}
Infants, children and adults are capable of extracting recurring patterns from their environment through statistical learning (SL), an implicit learning mechanism that is considered to have an important role in language acquisition. Research over the past 20 years has shown that $\mathrm{SL}$ is present from very early infancy and found in a variety of tasks and across modalities (e.g., auditory, visual), raising questions on the domain generality of SL. However, while $S L$ is well established for infants and adults, only little is known about its developmental trajectory during childhood, leaving two important questions unanswered: (1) Is SL an early-maturing capacity that is fully developed in infancy, or does it improve with age like other cognitive capacities (e.g., memory)? and (2) Will SL have similar developmental trajectories across modalities? Only few studies have looked at SL across development, with conflicting results: some find age-related improvements while others do not. Importantly, no study to date has examined auditory SL across childhood, nor compared it to visual SL to see if there are modality-based differences in the developmental trajectory of SL abilities. We addressed these issues by conducting a largescale study of children's performance on matching auditory and visual SL tasks across a wide age range (5-12y). Results show modality-based differences in the development of SL abilities: while children's learning in the visual domain improved with age, learning in the auditory domain did not change in the tested age range. We examine these findings in light of previous studies and discuss their implications for modality-based differences in $S L$ and for the role of auditory $S L$ in language acquisition. A video abstract of this article can be viewed at: https://www.youtube.com/watch?v=3kg35hoFOpw.
\end{abstract}

\section{RESEARCH HIGHLIGHTS}

- Is statistical learning (SL) an early-maturing capacity or does it improve with age? While SL has been studied extensively with infants and adults, very few studies examine the developmental trajectory of SL with children.

- We conducted a large-scale study of visual and auditory SL across childhood (ages 5-12).

- We find modality-based differences in the development of SL abilities: while children's visual SL improved with age, auditory SL did not change much across development.

- The findings point to modality-based differences in SL

\section{1 | INTRODUCTION}

One of the deepest questions in cognitive science is how children learn about the structure of their environment. One way of addressing this question is by examining children's learning mechanisms and, specifically, their ability to extract information about the world via statistical learning. Statistical learning (SL) refers to the ability to implicitly detect recurring patterns and regularities in sensory input based on their statistical properties and use this information to learn higher order structure, like that found in language (Thiessen \& Erickson, 2015). The term SL was originally coined in the domain of speech segmentation, where a seminal study showed that 
8-month-old infants are capable of using the transitional probabilities between syllables as a cue to word boundaries: after hearing an artificial language for a few minutes, infants could distinguish between words and foils based on their statistical properties (Saffran, Aslin, \& Newport, 1996).

$\mathrm{SL}$ is postulated to play an important role in language acquisition: it is offered as a way to explain how children learn about the structure of the language they are exposed to without having to assume much innate knowledge (Romberg \& Saffran, 2010). Indeed, SL was shown to facilitate learning across a wide range of linguistic domains (Saffran, 2003)-from learning phonemic inventories (Maye, Werker, \& Gerken, 2002) through word order preferences (Gervain, Nespor, Mazuka, Horie, \& Mehler, 2008), to the acquisition of syntactic patterns (Gómez \& Gerken, 1999). This body of literature illustrates learners' ability to extract linguistic structure by attending to distributional regularities in their environment. Supporting its role in language learning, individual differences in SL are predictive of various linguistic outcomes for both children (e.g., Arciuli \& Simpson, 2012; Kidd, 2012; Shafto, Conway, Field \& Houston, 2012) and adults (e.g., Conway, Bauernschmidt, Huang, \& Pisoni, 2010; Misyak \& Christiansen, 2012) .Taken together, these findings suggest that SL plays an important role in the process of language learning across the life span.

In the past two decades, numerous studies have shown that SL is present from early infancy (Kuhl, 2004; Saffran et al., 1996); plays a role in learning various types of statistical relations (Gebhart, Newport, \& Aslin, 2009); and is found across different modalities (i.e., auditory, visual and tactile domains; Conway \& Christiansen, 2005; Emberson, Conway, \& Christiansen, 2011). Surprisingly, while SL has been studied extensively in both infants and adults, very few studies have examined changes in SL from infancy to adulthood, despite the relevance of such findings for two fundamental questions on the nature of $S L$ and its role in learning. The first question has to do with the developmental trajectory of SL: Is SL an early-maturing capacity that does not change much throughout development, or does it improve with age? On the one hand, SL is already present in very young infants and postulated to play a role in language acquisition, suggesting that it is an early-maturing capacity. On the other hand, most other cognitive capacities do develop with age. The second question relates to the modality-specific characteristics of $\mathrm{SL}$. Although SL is found in multiple modalities and various sensory inputs, there is growing evidence for modality-based differences in adults' SL abilities (Frost, Armstrong, Siegelman, \& Christiansen, 2015; Krogh, Vlach, \& Johnson, 2012). If SL is a unitary mechanism, we may expect it to develop similarly across modalities. In contrast, a modality-specific mechanism may result in different developmental trajectories between modalities.

Interestingly, there is little data that can be brought to bear on these two questions. Only few studies have looked at how SL abilities change during development and they show a mixed pattern of results: while some argue that SL is age-invariant (e.g., Saffran, Newport, Aslin, Tunick, \& Barrueco, 1997), others report an improvement in SL with age (e.g., Arciuli \& Simpson, 2011, see detailed review in the next section). With regard to modality, while infants are capable of performing both auditory and visual SL (Bulf, Johnson, \& Valenza, 2011; Saffran et al., 1996), no study has compared children's SL abilities across modalities using similar tasks nor asked if performance is similarly affected by age in the two modalities.

In the current study we take a step towards filling this gap by conducting the first large-scale cross-sectional study of visual and auditory SL across childhood (ages 5 to 12, $N=230$ ), using two tasks that are matched on their statistical properties (learners are exposed to identical distributional input in the two modalities). Our main goal is to examine the effect of age on SL in the two modalities and the possible differences in developmental trajectory between them. In particular, we ask if the mixed pattern of results can be explained when modality is taken into account: does SL improve with age in the visual modality but not in the auditory one? We focus on the age range between 5 and 12 for theoretical and methodological reasons. This is the same age range for which there are contradictory findings on the effect of age in the visual and auditory domains (e.g., Arciuli \& Simpson, 2011; Saffran et al., 1997), yet no study has compared performance across the entire range. Looking at this age range allows us to test the hypothesis that age affects performance differently in the two modalities.

\section{1 | SL across development}

In theory, there are several possible predictions on the developmental trajectories of SL. The first is that SL improves with age, just like many other cognitive abilities (e.g., working memory, see Gathercole, Pickering, Ambridge, \& Wearing, 2004). This prediction is also motivated by recent findings from the field of implicit learning. $S L$ is often seen as a type of implicit learning (Perruchet \& Pacton, 2006), which was traditionally considered to be age-invariant (Reber, 1993). Yet this view has been challenged in recent years: while some studies support age-invariance (e.g., Meulemans, Van der Linden, \& Perruchet, 1998), there is growing evidence that implicit learning does improve with age (Janacsek, Fiser, \& Nemeth, 2012; Lukács \& Kemény, 2014; Vaidya, Huger, Howard, \& Howard, 2007). Since SL involves implicit learning, we may expect it to show a similar developmental trajectory and improve with age across modalities (Misyak, Goldstein, \& Christiansen, 2012).

A different prediction can be made when we consider the role of $\mathrm{SL}$ in language acquisition. Since infancy and early childhood are considered to be the prime-time for language learning (Birdsong, 1999), SL skills may be fully developed in infancy and not improve with age, a claim supported by the presence of SL in newborns (Bulf et al., 2011; Teinonen, Fellman, Näätänen, Alku, \& Huotilainen, 2009). Since language learning skills are claimed to deteriorate with age (Birdsong, 1999), SL skills may even decrease with age.

A third, more nuanced, prediction on the effect of age on SL takes into account modality-based differences. The fact that $\mathrm{SL}$ is found in multiple sensory modalities suggests that it is a domaingeneral mechanism that works similarly on different kinds of input, linguistic and nonlinguistic (Kirkham, Slemmer, \& Johnson, 2002; Saffran, Pollak, Seibel, \& Shkolnik, 2007; Saffran \& Thiessen, 2007). 
However, there is growing evidence of differences in learning between the auditory and visual domains that are more consistent with a modality-sensitive characterization of SL (Frost et al., 2015). On an individual level, performance on auditory and visual SL tasks is not correlated, indicating that they may tap onto different abilities (Siegelman \& Frost, 2015). There also seem to be qualitative differences in learning across modalities: adults showed better learning in the auditory domain when tasks were matched on statistical properties (Conway \& Christiansen, 2005; Saffran, 2002). The two modalities are affected differently by the same constraints: for instance, increasing stimuli presentation rate led to better learning in the auditory domain, but worse learning in the visual domain (Emberson et al., 2011). Such modality-based differences could arise if the processes involved in statistical learning (e.g., integration vs. extraction; Thiessen \& Erikson, 2013) are sensitive to the particular characteristics of the input (Frost et al., 2015).

From a developmental perspective, age may affect learning differently in the visual and auditory domains. Such a pattern has been reported in the domain of working memory where visual working memory improved more with age compared to audio working memory (Vountela et al., 2003). In particular, given its role in language acquisition, auditory SL may change less with age compared to visual SL. Language learning shows a unique developmental trajectory compared to other cognitive abilities whereby younger infants seem to show better capacities than older children and adults (Birdsong, 1999). Infants learn a great deal about language during their first year (e.g., Gervain \& Werker, 2008): if auditory SL plays a part in this process, we may expect it to be at its prime in early infancy and not to change much in later childhood. Visual processing, in contrast, seems to show a more linear developmental path, with older children showing better abilities than younger ones in tasks that require visual attention (e.g., Colombo, 2001; Scaife \& Bruner, 1975). Accordingly, we may expect visual SL to improve with age. Finding differences in the effect of age on learning in the two modalities would provide further support for the modality-sensitive nature of SL.

However, there is currently very little empirical evidence to support any of these predictions or distinguish between them. To date, only few studies have examined the effect of age on SL during childhood, and they exhibit a mixed pattern of results: while some studies find no difference between children and adults, others find an improvement with age. Interestingly, the difference seems to be, at least in part, modality related.

In the visual domain, two studies that compared children and adults on the same task found no effect of age. The first reports that children (aged 9 to 12) performed similarly to adults on a visual SL task (Bertels, Boursain, Destrebecqz, \& Gaillard, 2015). The second found that adults performed significantly better on a visual SL task compared to children (age 6 to 11) and adolescents (age 12 to 17), but reports no significant difference between children and adolescents despite developmental changes to the hippocampal structure (Schlichting, Guarino, Schapiro, Turk-Browne, \& Preston, 2017). Overall, these findings seem to point to age-invariance in visual SL across childhood. However, both studies are based on quite small samples, examine a relatively narrow slice of development and look at performance at a few age points rather than across a wide age range-all of which may mask the effect of age on performance (Arciuli \& von Koss Torkildsen, 2012; Bertals et al., 2015). A more comprehensive study of visual SL across development did find clear age-related improvement: Arciuli and Simpson (2011) examined visual SL in 183 children between the ages of 5 and 12 and found that older children showed significantly better abilities. This single comprehensive study suggests that visual SL does in fact improve with age.

There is even less work that examined SL across development in the auditory domain, despite its postulated role in language acquisition. Only one study compared children and adults on the same auditory task. Saffran et al. (1997) found no difference in auditory SL between 6-year-olds and undergraduate students, with both age groups showing similar learning. This is somewhat surprising given that auditory artificial grammar learning improves with age, with adults showing better learning than 6- and 9-year-olds (Saffran, 2001).

In sum, while Arciuli and Simpson's (2011) findings suggest that visual SL improves during development, the only study to compare auditory SL in children and adults found no difference between them. One possibility is that age does affect SL in the auditory modality as well, but that this pattern is not detected when using relatively small samples and comparing only one age group to adults (as done in the study reported above). Alternatively, it is possible that there are fundamental differences between visual and auditory $S L$ that are also reflected in different developmental trajectories in the two modalities. In particular, SL may improve more slowly (or less) in the auditory domain. These possibilities are hard to evaluate given the existing literature since all the studies that have compared performance in the auditory and visual domain involve adults. Moreover, no study to date has compared auditory and visual learning in children across a wide age range using tasks with similar distributional properties.

\section{2 | THE CURRENT STUDY}

To test the three different predictions on the effect of age on SL, we conducted the first large-scale study of children's performance on auditory and visual SL across a wide age range (5-12y). We ask whether performance in these tasks is affected by age, and if so, then how: will auditory SL improve across development as found in the visual domain, or will SL have different developmental trajectories in the two modalities? Our study is a first step in addressing the paucity of data on the developmental trajectory of SL. It aims to provide crucial and novel findings on two important questions: (1) the effect of age on SL during childhood; and (2) the modality-sensitive nature of SL. Our study extends previous work by conducting the first large-scale study of auditory and visual SL during childhood using tasks that have the same distributional structure.

In order to examine children's SL abilities in both domains, we conducted two studies: Experiment 1 tested auditory SL (ASL) and Experiment 2 tested visual SL (VSL). We first report the results of the two experiments separately, and then compare them using a unified 
analysis. Importantly, while there are differences between the tasks in that one uses auditory stimuli (syllables) and the other visual stimuli (alien shapes), they are matched on several important properties. First, the tasks have the same statistical properties for items during exposure and testing: same number of triplets, same TPs within items and between items, same number of test trials and identical construction of foils. This makes the statistical structure of the input identical between modalities. Second, the tasks were run using the same procedure, with a similar cover story by the same research assistants in the same environmental setting. Both factors increase our ability to compare performance in the two modalities.

We focus on the ages between 5 and 12 for several reasons. Theoretically, our main goal is to ask if age affects performance differently in the two modalities. The chosen age range is the one most suited to address this question because this is the same range for which there are contradictory findings in the visual and auditory domains (Arciuli \& Simpson, 2011; Saffran et al., 1997), yet there is no study that looked at both modalities with these ages using tasks with similar distributional properties. Methodologically, while there is also need for developmental data from infancy to childhood, there are currently no existing tasks that can be reliably used with this entire sample. Qualitatively and quantitatively different paradigms are used to measure learning in infants compared to older children and adults. Studies of SL in infancy use implicit measure like gaze duration and head turning, which are less reliable with more mobile older children and adults (Cristia, Seidl, Singh, \& Houston, 2016). Most studies with adults use explicit judgments, which cannot be used successfully with young children or infants. Consequently, there is a real methodological challenge in collecting the whole range of needed developmental data (see also the General Discussion). While the current study does not cover the entire age range from infancy to late childhood, it provides crucial and novel findings on the developmental trajectory of SL.

\section{3 | GENERAL METHOD}

Our auditory task was closely modeled on the classic segmentation task developed by Saffran et al. (1996), and our visual task closely resembled the child-friendly task used in Arciuli and Simpson (2011), based on Turk-Brown, Jungé, and Scholl (2005). As mentioned above, the two tasks were matched on several important properties to enable better comparison between them. We describe the tasks in detail in the method section of each experiment. While the tasks differed in the stimuli used (syllables vs. alien shapes), they were comparable in terms of the distributional information learners are exposed to.

\section{1 | Participants}

In all, 115 children participated in the auditory task reported in Experiment 1 (age range: 5-12y, mean age: 8:4y, 65 boys and 50 girls) and 115 children participated in the visual task reported in Experiment 2 (age range: $5-12 y$, mean age: $8: 3 y, 60$ boys and 55 girls). All children were visitors at the Bloomfield Science Museum in Jerusalem and were recruited for this study as part of their visit to the Israeli Living Lab. All participants received a small educational reward in return for participation. Parental consent was obtained for all children participating in both experiments. All of the children who participated in the auditory task (Experiment 1) were native speakers of Hebrew, as were the majority of the children participating in the visual task (Experiment 2). None of the children had known language or learning disabilities.

\section{4 | EXPERIMENT 1: ASL}

\section{1 | Materials}

The auditory stimuli consisted of a synthesized "alien" language, containing five unique tri-syllabic words (made up of 15 different syllables): "dukame", "gedino", "kimuga", "nalobi" and "tobelu". All words were synthesized using the PRAAT software in order to control for syllable duration and frequency. Average word length was $850 \mathrm{~ms}$. The words were concatenated together in a semi-randomized order (with the constraint that no word would appear twice in a row) to create an auditory familiarization stream. In this stream, the transitional probabilities (TPs) between syllables within a word were always 1, while the TPs between words were 0.25 (because syllables were not repeated across words and because each word could be followed by any of the other four). The exposure phase lasted 2;20 minutes, with each word repeated 32 times. Importantly, there were no breaks between words and no prosodic or co-articulation cues in the stream to indicate word boundaries.

The test phase included 25 two alternative forced-choice trials (2AFC), in which participants heard two possible "words" (separated by $500 \mathrm{~ms}$ ), and had to choose which one sounded more like the language they had just heard. On each trial, participants heard a real word (like "dukame") either followed or preceded by a foil word. Foil words were constructed by taking the first syllable from one word, followed by the second syllable from another word, and the third syllable from a third word. Thus, each syllable in the foil words appeared in a similar position in real words, but with different surrounding syllables (for example, "kilome" or "dubega"). This created a difference in the statistical properties of the words and foils: while the TPs between every two adjacent syllables within a word are 1, the TPs between every two syllables in a foil test item are 0 , as participants never heard these syllables one after the other during familiarization. If participants learn the statistical properties of the syllables in the stream, they should be able to distinguish between words and foils. The possible score on this task ranged from $0 \%$ accuracy $(0 / 25$ trials correct) to $100 \%(25 / 25$ trials correct).

We assessed learning by comparing responses to words and nonwords, rather than part words, for two reasons. The main reason was that we wanted to avoid floor effects in learning. Because our exposure time was much shorter than previous ASL studies with children (under 3 minutes compared to over 20 minutes in Saffran et al., 1997), we wanted to use more salient distinctions to assess learning. Since our goal was not to show that children can discriminate words from part words (a finding shown in other studies), but to see how that ability changes with age, 
FIGURE 1 Accuracy in the task by age (in half years). Each dot represents an accuracy score (ranging from $0 \%$ to $100 \%$ ) shown by one or more of the participants in the relevant age range. The size of the dot reflects the number of participants that had that score. For example, two 8-yearolds were $60 \%$ accurate while one was $80 \%$ accurate. The plotted blue line represents the linear regression line, with the standard confidence interval in gray. The black line represents the $50 \%$ chance level

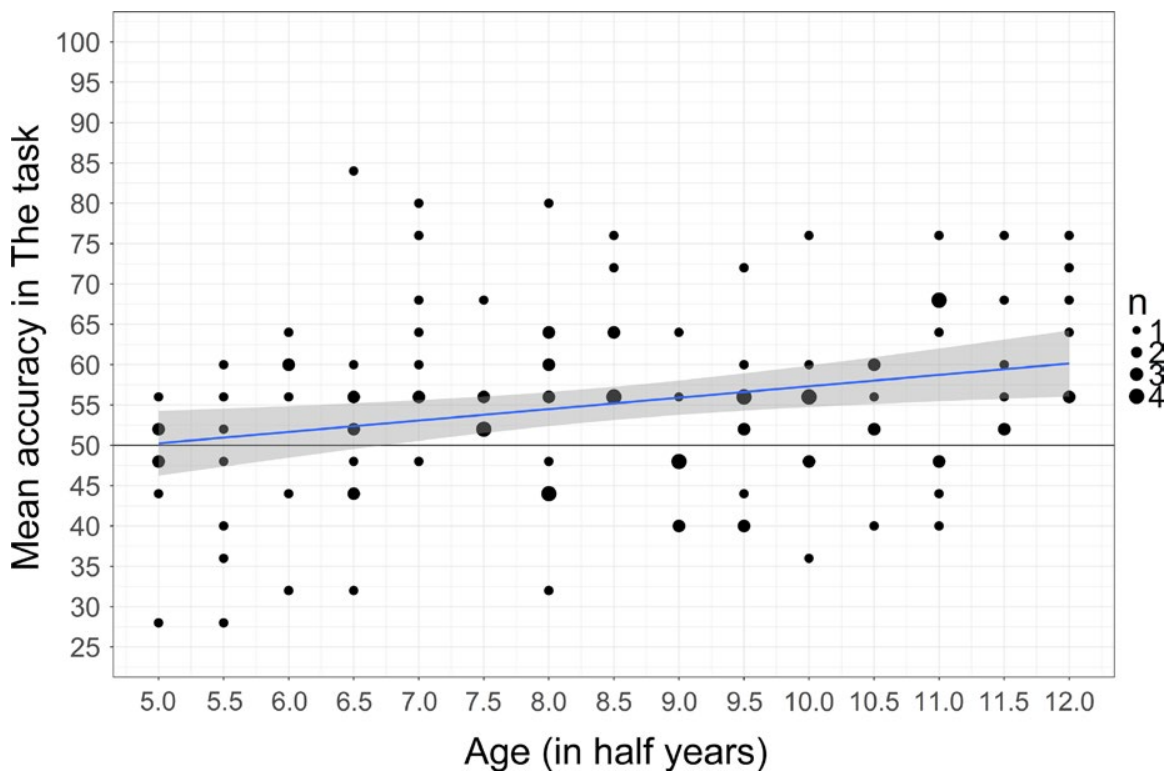

.0001). Figure 1 shows children's mean performance on the task as a function of age: there seems to be an effect of age on performance, with older children showing better accuracy. To test for significance, we used mixed-effect logistic regression models. Our dependent binominal variable was success in a single test trial. The model included fixed effects for age (in half years), trial number (centered), order of appearance in the test (word-first trials vs. general mean, deviation coding ${ }^{1}$ ) and gender (females vs. general mean, deviation coding). Following Barr, Levy, Scheepers, and Tily (2013), the model had the maximal random effect structure justified by the data that would converge. The model had random intercepts for participants and items and by-participants slopes for the effect of trial number (see Table 1).

Age had a significant positive effect on performance: children's accuracy in the auditory task improved with age, with older children showing higher accuracy $(\beta=0.056, S E=0.02, p<.01)$. The effect of trial number was not significant $(\beta=-0.004, S E=0.005, p>.1$ ), confirming that no learning (or unlearning) was happening during the test phase itself, despite the repetition of both foils and words. The effect of gender was also not significant $(\beta=-0.04, S E=0.04, p>.1)$. Interestingly, order of appearance in the test significantly affected performance, with better accuracy on word-first trials ( $\beta=0.15, S E=0.03$, $p<.001)$. Children were better in trials where the real word was heard before the foil: since the order of presentation was counter-balanced

TABLE 1 Regression model for auditory SL (ages 5-12)

\begin{tabular}{lllll} 
& Estimate & Std. Error & $z$ value & $p$-value \\
\hline (Intercept) & 0.199 & 0.042689 & 4.662 & $0.000^{* * *}$ \\
\hline Age & 0.056501 & 0.020871 & 2.707 & $0.006^{* * *}$ \\
\hline Trial Number & -0.00418 & 0.005416 & -0.771 & 0.44 \\
\hline $\begin{array}{l}\text { Order of } \\
\text { Appearance }\end{array}$ & 0.159547 & 0.038171 & 4.18 & $0.000^{* * *}$ \\
\hline Gender & -0.04061 & 0.042598 & -0.953 & 0.34 \\
\hline
\end{tabular}




\begin{tabular}{lcllll} 
& N & $\begin{array}{l}\text { Mean ASL } \\
\text { score }\end{array}$ & $\begin{array}{l}\text { Std. } \\
\text { Deviation }\end{array}$ & t-value & $p$-value \\
\hline Age group 5 to 6 & 20 & $48 \%$ & 2.4 & $t(19)=-0.74$ & 0.465 \\
\hline Age group 6.5 to 7.5 & 24 & $57 \%$ & 2.4 & $t(23)=2.98$ & $0.006^{* *}$ \\
\hline Age group 8 to 9 & 26 & $55 \%$ & 2.2 & $t(25)=2.37$ & $0.025^{*}$ \\
\hline Age group 9.5 to 10.5 & 24 & $53 \%$ & 1.9 & $t(23)=1.8$ & 0.084 \\
\hline Age group 11 to 12 & 21 & $60 \%$ & 2.3 & $t(20)=4.61$ & $0.000^{* * *}$ \\
\hline All children & 115 & $55 \%$ & 1 & $t(114)=4.79$ & $0.000^{* * *}$ \\
\hline
\end{tabular}

TABLE 2 Auditory SL accuracy by age bins this could not reflect a preference for pressing 1 or 2 . The advantage for word-first trials is in line with the "interval bias" which is often found in 2AFC tests (see García-Pérez \& Alcalá-Quintana, 2010, 2011; Yeshurun, Carrasco, \& Maloney, 2008, for reviews).

These results suggest that auditory SL improves with age. However, a somewhat different pattern emerged when we examined participants' performance in age bins: We divided our sample into five age bins each spanning exactly one year to look more closely at performance across development (the resulting bins had a relatively similar number of participants in each bin and across tasks). Importantly, we used one-year age bins only for presentational clarity: age was entered as a continuous factor (in half years), without any division into bins, in all analyses. When breaking down the results by one-year age bins, we saw that children in the youngest age group (ages 5 to 6) did not show learning: while all other age groups were significantly better than chance, the performance of the youngest group did not differ from chance $(M=48 \%, t(19)=-0.74, S E=2.4, p$ $>.1$, see Table 2). This is also reflected in Figure 1, which shows that the majority of children aged 6 and below are performing at chance level. We therefore conducted a second analysis to see if the effect of age on performance was driven by the inclusion of the youngest age group that showed no learning.

We ran an additional mixed-effect model with a similar effect structure using the data obtained only from children older than 6 (without the 20 children in the youngest age bin, Table 3). The model had the same fixed and random effects structure as the model reported in Table 1. As suspected, the effect of age disappeared in the new model (Table 3): without the youngest children, age was no longer predicative of accuracy $(\beta=0.02, S E=0.02, p>.1)$. That is, auditory SL did not show an improvement between the ages of 6.5 and 12, a significant developmental window.

TABLE 3 Regression model for auditory SL (ages 6.5-12)

\begin{tabular}{|lllll|} 
& Estimate & Std. Error & $\mathrm{z}$ value & $p$-value \\
\hline (Intercept) & 0.26018 & 0.047032 & 5.532 & $0.000^{* * *}$ \\
\hline Age & 0.02571 & 0.028043 & 0.917 & 0.359 \\
\hline Trial Number & -0.00633 & 0.006127 & -1.034 & 0.301 \\
\hline $\begin{array}{l}\text { Order of } \\
\text { Appearance }\end{array}$ & 0.15576 & 0.042117 & 3.698 & $0.000^{* * *}$ \\
\hline Gender & -0.03824 & 0.047029 & -0.813 & 0.41 \\
\hline
\end{tabular}

To further support the importance of this null result, we ran a Bayes Factor analysis for the correlation between age and performance on the auditory task. This analysis allows us to compare the likelihood probability of two competing hypotheses (null hypothesis: no effect of age vs. alternative: effect of age) rather than just provide evidence against the null hypothesis. When all children were included in this analysis, we found moderate support for correlation between age and performance $(r=0.25, \mathrm{BF}=4.9)$. However, the opposite trend emerged when excluding the youngest age group of children: without them, there was actually moderate support for the null hypothesis ( $r$ $=0.09, \mathrm{BF}=0.2$ ), strengthening the claim that there isn't a significant correlation between age and performance in the auditory task after age 6.

This finding matches previous reports of age-invariance in the auditory domain (Saffran et al., 1997) and contrasts with reports from the visual domain (Arciuli \& Simpson, 2011). It suggests that (1) auditory SL is a rather stable, age-invariant capacity during childhood, and (b) that there are modality-based differences in the development of SL. Importantly, the fact that children did not show learning in the youngest age group does not mean that they are incapable of learning auditory regularities (a pattern that would be inconsistent with the findings of auditory SL in infancy, see Saffran et al., 1996), but may simply reflect their difficulty with the more explicit measure of learning used here (we expand on this in the discussion). Nevertheless, the lack of change in performance once learning is shown (between ages 6.5 and 12) suggests that auditory SL does not improve during childhood. To further explore these differences, we conducted a second study looking at the development of visual SL across the same age range (5 to 12) and using a task that was matched on the statistical properties of the input.

\section{5 | EXPERIMENT 2: VISUAL SL}

\section{$5.1 \mid$ Materials}

The visual stimuli consisted of a continuous temporal stream of colorful alien figures, containing five unique triplets of aliens (a total of 15 different aliens). Each alien figure appeared on the screen for $500 \mathrm{~ms}$, with a 100 ms break between figures-resulting in a 1800 ms presentation time for each triplet. The triplets were created anew for each participant, so each participant saw a different set of triplets. For each 
FIGURE 2 Accuracy in the visual task by age (in half years). Each dot represents an accuracy score (ranging from $0 \%$ to $100 \%)$ shown by one or more of the participants in the relevant age range. The size of the dot reflects the number of participants who had that score. For example, one 7 -year-old was $60 \%$ accurate while another was $40 \%$ accurate. The plotted blue line represents the linear regression line, with the standard confidence interval appearing in gray. The black line represents the $50 \%$ chance level

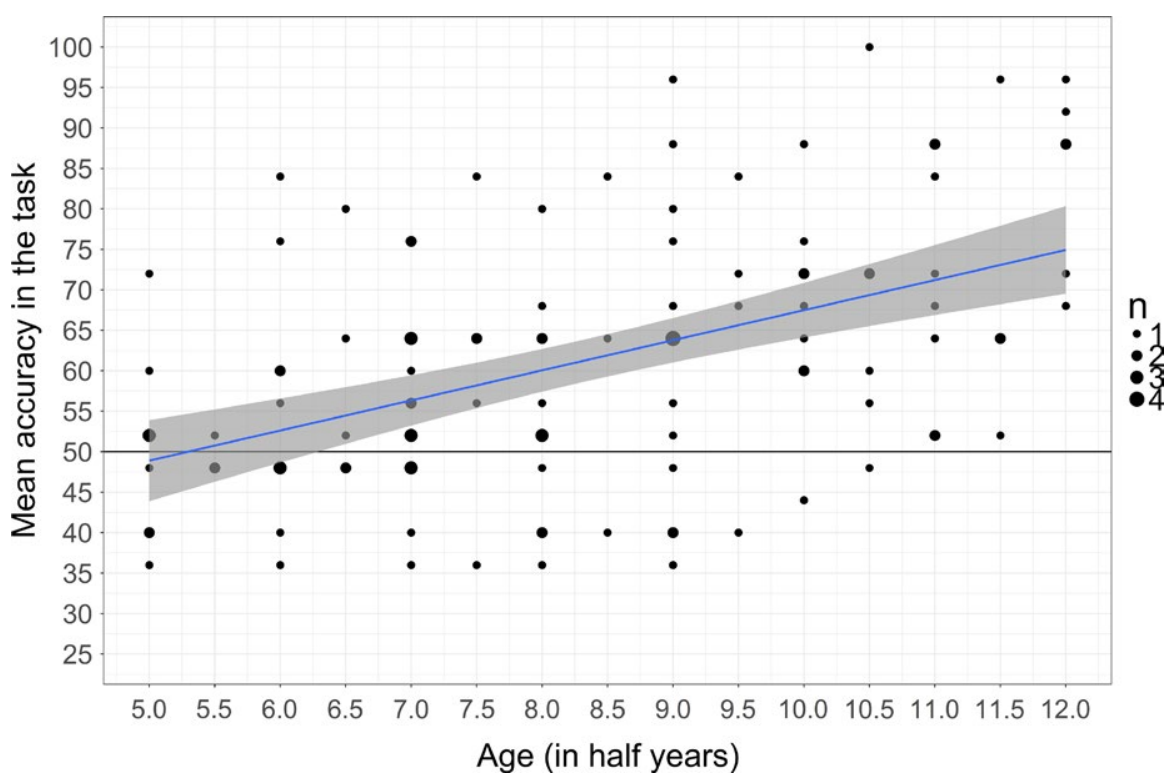

participant, the five triplets were concatenated together in a semirandomized order (with the constraint that no triplet would appear twice in a row) to create the visual familiarization stream. The TPs between aliens within a triplet was 1 , while the TPs of aliens between triplets was 0.25 . The exposure phase lasted 3:30 minutes, with each triplet repeated 24 times. Importantly, there were no visual or temporal cues to indicate triplet boundaries.

The test phrase included 25 two alternative forced-choice trials (2AFC), in which participants saw two triplets and had to choose which three aliens really appeared in the same order in the stream they just saw. On each trial, participants saw a real triplet of aliens, either followed by or preceded by a foil triplet. Similar to the auditory task, foil triplets were constructed by combining aliens from different triplets, so that each alien in the foil triplets appeared in a similar position in real triplets, but with different surrounding aliens. This created a difference in the statistical properties of the triplets and foils: while the TPs between every two adjacent aliens within a triplet are 1, the TPs between every two aliens in a foil triplet are 0 , as participants never saw these aliens appearing one after the other during familiarization. If participants are learning the statistical properties of the aliens in the visual stream, they should be able to distinguish between triplets and foils.

\section{2 | Procedure}

Children were told that they were about to see a aliens walking into a spaceship, and were then exposed to the familiarization stream on the screen while hearing calming electronic music through soundcancelling headphones (this was done to increase the similarity in the procedure of the two studies and isolate children from distracting ambient noise). Following exposure, children were asked to help the spaceship commander who doesn't remember which aliens walked in together: they were asked to say which of the two triplets they will see walked into the spaceship together.
The 25 2AFC test trials were presented to children in random order (with the constraint that the same triplet/foil would not appear in two consecutive trials). The order of triplets and foils on each trial was counter-balanced so that in half of the trials the real triplet appeared first while in the other half the foil triplet appeared first. Children saw the two triplets sequentially. After seeing both possibilities, children were asked to press either "1" or "2" according to whether they thought the correct triplet was the first or the second they saw. In cases where children felt that they didn't know the answer, the experimenter encouraged them to try and guess what looked more familiar according to the aliens they saw. At the end of the task, the experimenter thanked the child for helping the spaceship commander.

\section{3 | Experiment 2: Results}

As a group, children showed significant learning in the visual task: their mean accuracy score was $61 \%$, which is significantly above chance $(t(114)=7.56, S E=15, p<.0001)$. Figure 2 shows children's mean performance on the task as a function of age (in half years): age seems to affect performance, with older children showing better accuracy. To test for significance, we used mixed-effect logistic regression models, as we did for the auditory task. Our dependent binominal variable was success in a single test- trial. The model included fixed effects for age (in half years), and trial number (centered), order of appearance in the test (triplet-first general mean, deviation coding) and gender (females vs. general mean, deviation coding). The model had the maximal random effects structure justified by the data that would converge, including random intercepts for participants but not for items, as these were unique for each participant. We also included by-participant slopes for the effects of trials number and order of appearance (see Table 4).

Age had a strong, positive effect on performance, with children's accuracy significantly improving with age $(\beta=0.16, S E=0.03$, 
TAB LE 4 Regression model for visual SL (ages 5-12)

\begin{tabular}{|lllll|} 
& Estimate & Std. Error & $z$ value & $p$-value \\
\hline (Intercept) & 0.501826 & 0.0612029 & 8.199 & $0.000^{* * *}$ \\
\hline Age & 0.167948 & 0.0301024 & 5.579 & $0.000^{* * *}$ \\
\hline Trial Number & 0.000124 & 0.0059624 & 0.021 & 0.983 \\
\hline $\begin{array}{l}\text { Order of } \\
\text { Appearance }\end{array}$ & 0.046148 & 0.0410112 & 1.125 & 0.26 \\
\hline Gender & -0.05012 & 0.0605512 & -0.828 & 0.408 \\
\hline
\end{tabular}

$p<.001)$. The effect of trial number was not significant $(\beta=0.0001$, $S E=0.005, p>.1$ ), confirming that no learning (or unlearning) was happening during the test despite the repetitions of both foils and triplets. The effect of gender was also not significant $(\beta=-0.05, S E=0.06$, $p>$.1). Interestingly, there was no effect for the order of appearance in the test: unlike in the auditory task children did not show an interval bias: they were not better when the real triplet appeared before the foil $(\beta=0.04, S E=0.04, p>.1)$

Given the pattern we found in the auditory domain, we wanted to look more closely at performance in the different age bins. We divided participants into the same descriptive one-year age bins used in the auditory task (Table 5). We discovered that as in Experiment 1, the youngest age group (children aged 5 to 6) did not show significant learning on the task; their performance did not differ from chance $(M$ $=52.5 \%, t(21)=-0.96, S E=2.6, p>.1)$, while all other age groups performed above chance (see Table 5). As in Experiment 1, we wanted to see if the effect of age was driven by the inclusion of the youngest age group that showed no learning-although this seemed less likely in the visual domain given the larger coefficient of age in the model.

We therefore conducted a second analysis without the children between the ages of 5 and $6(N=22)$. The model had the same fixed effects and random effects structure as the model reported in Table 6. Importantly, unlike in the auditory domain, the effect of age remained significant after excluding the youngest age group: children showed a strong improvement with age in the revised model $(\beta=0.2, S E=$ $0.04, p<.001)$. That is, visual SL, unlike auditory SL, does show an improvement between the ages of 6.5 to 12 . This finding confirms previous reports of an improvement in visual SL across childhood (Arciuli \& Simpson, 2011) and points to interesting developmental differences between SL in the visual and auditory domain. To further explore the relation between age and performance, we ran a Bayes Factor analysis
TABLE 6 Regression model for visual SL (ages 6.5-12)

\begin{tabular}{lllll} 
& Estimate & Std. Error & $z$ value & $p$-value \\
\hline (Intercept) & 0.601812 & 0.072092 & 8.348 & $0.000^{* * *}$ \\
\hline Age & 0.202317 & 0.042575 & 4.752 & $0.000^{* * *}$ \\
\hline Trial Number & 0.00428 & 0.007006 & 0.611 & 0.541 \\
\hline $\begin{array}{l}\text { Order of } \\
\text { Appearance }\end{array}$ & 0.014828 & 0.047458 & 0.312 & 0.755 \\
\hline Gender & -0.104 & 0.07274 & -1.429 & 0.153 \\
\hline
\end{tabular}

(as in the auditory domain) and found that in contrast with the results from the auditory domain, in the visual task there was very strong support for a positive correlation between age and performance, regardless of whether the youngest group of children was included in the analysis $(r=0.47, \mathrm{BF}>1000)$ or excluded from it $(r=0.43$, BF > 1000). To further explore these differences between modalities, we conducted an analysis of the combined data from the two studies.

\section{6 | COMPARING AUDITORY AND VISUAL SL}

Since the auditory and visual modalities seem to display different developmental trajectories, we further explored the differences between them by directly comparing children's performance in both experiments. More specifically, we wanted to compare the effect of age on performance on the two tasks between the ages of 6.5 and 12, excluding the youngest group of children who did not show significant learning in either task $(N=42)$. To do this, we ran a mixed-effect logistic regression on the combined dataset $(N=188)$. The dependent binomial variable was accuracy on a given trial. The model included fixed effects for age (in half years, as a continuous and centered factor), modality (visual vs. auditory, dummy coded), order of appearance (wordfirst trials vs. general mean, deviation coding) and the interaction terms modality $\times$ age and modality $\times$ order of appearance (Table 7 ). The model had the maximal random effect structure justified by the data that would converge, including random intercepts for participants and a by-participant slope for the effect of order of appearance.

As expected, the effect of age was not significant in the combined model $(\beta=0.02, S E=0.03, p>.1)$, but the interaction between age and modality was highly significant $(\beta=0.13, S E=0.05, p<.01)$. This

\begin{tabular}{|c|c|c|c|c|c|}
\hline & $\mathrm{N}$ & $\begin{array}{l}\text { Mean } \\
\text { VSL } \\
\text { score }\end{array}$ & $\begin{array}{l}\text { Std. } \\
\text { Deviation }\end{array}$ & $\mathrm{t}$-value & $\mathrm{p}$-value \\
\hline Age Group 5 to 6 & 22 & $52 \%$ & 2.6 & $t(21)=-0.96$ & 0.347 \\
\hline Age Group 6.5 to 7.5 & 26 & $57 \%$ & 2.4 & $t(25)=2.92$ & $0.007^{* *}$ \\
\hline Age Group 8 to 9 & 30 & $59 \%$ & 2.9 & $t(29)=3.1$ & $0.004^{* *}$ \\
\hline Age Group 9.5 to 10.5 & 19 & $67 \%$ & 3.3 & $t(18)=5.06$ & $0.000^{* * *}$ \\
\hline Age Group 11 to 12 & 18 & $74 \%$ & 3.6 & $t(17)=6.88$ & $0.000^{* * *}$ \\
\hline All Children & 115 & $61 \%$ & 1.4 & $t(114)=7.56$ & $0.000^{* * *}$ \\
\hline
\end{tabular}

TABLE 5 Visual SL accuracy by age bins 
TABLE 7 Regression model for visual and auditory SL (ages 6.5-12)

\begin{tabular}{|lllll} 
& Estimate & Std. Error & z-value & $p$-value \\
\hline (Intercept) & 0.26758 & 0.05559 & 4.814 & $0.000^{* * *}$ \\
\hline Modality (Visual vs. Auditory) & 0.2536 & 0.08206 & 3.09 & $0.001^{* *}$ \\
\hline Age & 0.02355 & 0.03291 & 0.716 & 0.474 \\
\hline Order of Appearance & 0.16103 & 0.04242 & 3.796 & $0.000^{* * *}$ \\
\hline Modality X Age & 0.13335 & 0.05152 & 2.588 & $0.009^{* *}$ \\
\hline Modality X Order of Appearance & -0.14315 & 0.063 & -2.272 & $0.023^{*}$
\end{tabular}

suggests that an increase in participant's age was associated with improved performance in the visual SL task, but not in the auditory SL task. This result matches our observation from Experiments 1 and 2: age-related effects in SL were found only in the visual domain. The model also showed a significant main effect of modality $(\beta=0.25$, SE $=0.08, p<.01$ ), with visual SL being better than auditory SL across childhood. This modality-based difference in the development of SL is illustrated in Figure 3, which shows that visual learning improves with age, while auditory learning does not. Interestingly, an additional difference between the modalities is seen in the effect of order-ofappearance during testing: performance was better overall when the real word/triplet appeared first ( $\beta=0.16, S E=0.04, p<.001$ ), but this was qualified by a significant interaction with modality $(\beta=-0.14, S E$ $=0.06, p<.05)$, indicating that the interval bias was only affecting performance in the auditory modality.

\section{7 | DISCUSSION}

We set out to explore the effect of age on visual and auditory SL throughout childhood as a way of better understanding the nature of $\mathrm{SL}$ and its role in learning. While SL has been studied extensively over the past 20 years, very few studies have examined SL during development, and they report mixed findings. While some suggest that SL improves with age (e.g., Arciuli \& Simpson, 2011), others report that it is age-invariant (Saffran et al., 1997). In this paper, we wanted to contrast three different predictions on the effect of age on SL: the first is that $S L$ is age-invariant and does not change much across development; the second suggests that SL improves with age, but that this pattern can only be detected by looking across a large slice of development with a big enough sample; the third proposes that the effect of age on SL is modality-based: visual SL will improve with age while auditory SL will not. To evaluate these predictions, we conducted a large-scale study of visual and auditory SL across childhood using tasks that were matched on their statistical properties.

Our results provide clear support for the third prediction: we found a contrast between the developmental trajectories of SL abilities in the auditory and visual modalities: While visual SL improved significantly during childhood (between the ages of 6.5 and 12), auditory SL did not. These results mirror the findings of Arciuli and Simpson (2011), who reported an improvement with age in the visual domain for similar ages, and are also in line with Saffran et al. (1997), who reported age-invariance in the auditory domain. When taking modality into account, the existing findings are no longer contradictory. Instead, they suggest that there are important modality-based differences in children's statistical learning abilities: age affected development differently in the two domains, and learning was better in the visual domain compared to the auditory one.

The finding that auditory SL does not improve with age is supportive of Reber's claim that some implicit learning mechanisms-especially those used early in life-are age-invariant. The age-invariance seen in this study is consistent with the postulated role of auditory SL in language acquisition (e.g., Saffran et al., 1996; Saffran et al., 1997), which
FIGURE 3 Accuracy in both SL tasks by modality and age (in half years). Each dot represents an accuracy score (ranging from $0 \%$ to $100 \%$ ) shown by one or more of the participants in the relevant age range. The size of the dot reflects the number of participants who had that score. The two colorful plotted lines represent the linear regression lines for each modality, with the standard confidence interval appearing in gray. The black line represents the $50 \%$ chance level

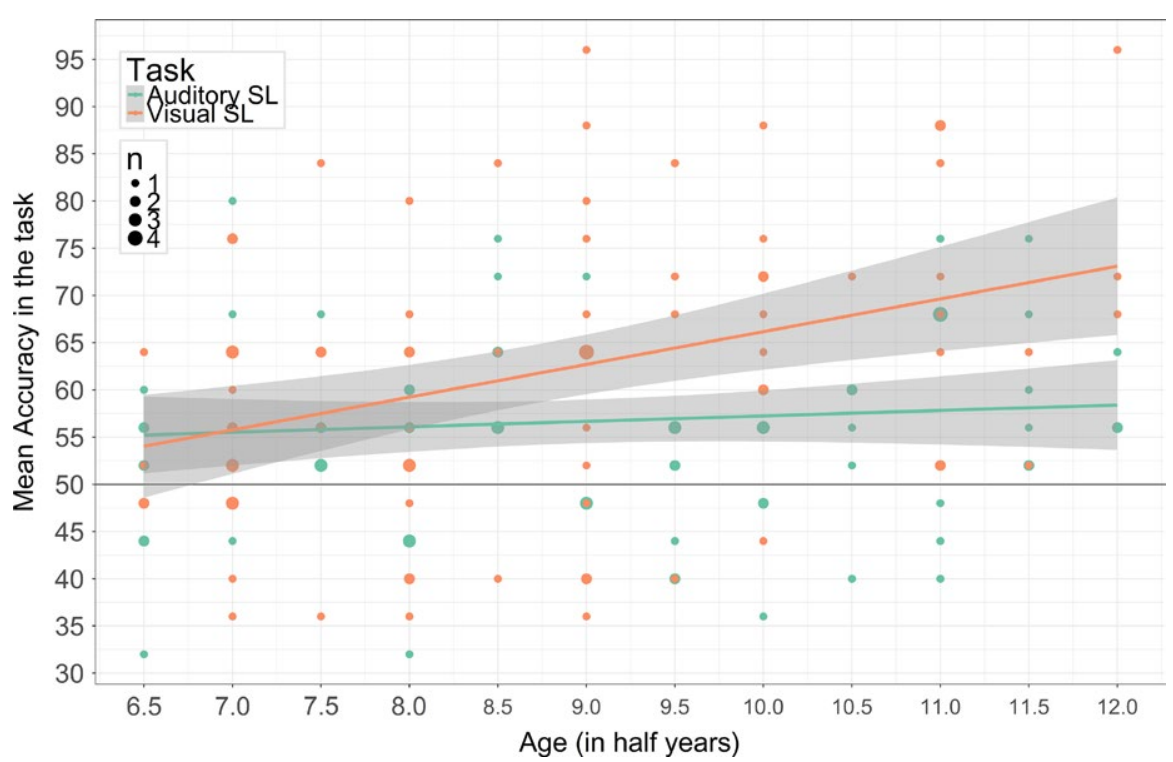


is often argued to be at its prime during infancy and early childhood (Birdsong, 1999). Finding that auditory SL does not improve during childhood supports its role in language acquisition (Arciuli \& von Koss Torkildsen, 2012; Romberg \& Saffran, 2010) by showing that it is an early-maturing capacity already developed at a time when children need to discover regularities and structure in their native language. However, this conclusion is limited: until we collect data on auditory $\mathrm{SL}$ at the age range between 8 months (for which there is ample evidence of auditory SL; e.g., Saffran et al., 1996) and 6 years of age, we cannot know if auditory SL abilities change or remain stable between birth and age 6. As discussed in the introduction, no study to date has looked across these ages, among other things because of the methodological challenge in devising tasks that can be used appropriately and reliably across the entire age range.

Findings from the period between infancy and early childhood are crucial in understanding the developmental trajectory of auditory SL: this is the time period in which children's language skills develop the most, and in which many other cognitive changes occur. The lack of data from this age range means that we cannot distinguish between several different developmental accounts of SL. On the one hand, auditory SL could be fully developed from birth and not change much throughout late infancy. Such an account would be consistent with arguments of invariant implicit learning mechanisms. Alternatively, SL could gradually improve between infancy and early childhood, parallel to other cognitive changes in this time period. This account is supported by the findings of Emberson et al. (under review) who found better auditory SL in 10-month-old infants compared to 8-month-old infants. A third possibility is that auditory SL improves significantly during the first year of life, as infants learn about the sounds of their native language (Kuhl, 2004), and then remains constant throughout development or changes its efficiency due to prior knowledge and experience with specific types of regularities (Thiessen, Girard, \& Erickson, 2016). This view is supported by studies showing that prior linguistic knowledge can mediate word segmentation in both adults and infants (Finn \& Hudson-Kam, 2008; Thiessen \& Saffran, 2003). In order to distinguish between these different trajectories, one needs an auditory SL task that can be used with children from early infancy to childhood (and such tasks do not currently exist).

In both tasks, the youngest age group (ages 5-6y) did not show learning: they were not significantly above chance. While it is theoretically possible that this pattern reflects a developmental change, several factors point against such an interpretation. There are several reasons to believe that this pattern does not indicate a qualitative change in learning after age 6 (or a so-called "step function") but instead reflects young children's difficulty with the relatively explicit judgments required in the task (e.g., saying whether the sounds make up a real word or not). First, infants do exhibit learning in both visual and auditory SL when using more implicit measures like listening times (e.g., Kirkham et al., 2002; Saffran et al., 1996) and SL was found already in newborns (e.g., Bulf et al., 2011; Teinonen et al., 2009). Developmentally, it is unlikely that an ability that is present early in life disappears before age 6 only to resurface again 6 months later. A more likely explanation is that the youngest children were learning the regularities but failed to manifest this implicit knowledge using the explicit forced-choice task used in this study. Supportive of this claim, younger children generally struggle in learning artificial languages in laboratory settings, resulting in worse performance overall (Ferman \& Karni, 2010; Folia, Uddén, De Vries, Forkstam, \& Petersson, 2010). That is, the lack of learning in the youngest age group seems more consistent with difficulty in performing the explicit task than with a change in SL.

Further work is needed to see if indeed there is a change in SL abilities during early childhood. To conduct such work, we need to develop more implicit tasks that can be used with younger age groups. More implicit measurements (e.g., reaction times) have been recently put to use with adult learners (self-paced visual presentation; Karuza, Farmer, Fine, Smith, \& Jaeger, 2014; Rapid Serial Auditory Presentation; Franco, Eberlen, Destrebecqz, Cleeremans, \& Bertels, 2015). However, these tasks are not suitable for assessing auditory learning and, more importantly, are not suitable for use with infants, and are challenging to use with young children, who exhibit a greater degree of variance. Since there are large age-related and individual differences in motor control during early childhood, it is problematic to use RTs to compare performance across different ages. We are currently working on developing novel implicit SL tasks that could be used with both infants and young children to give a more comprehensive description of the developmental trajectory of SL during early childhood.

\section{1 | Modality-based differences in SL}

There are multiple factors that may lead to differences in performance in the auditory and the visual domain: the stimuli in the two tasks are inherently different, as is our accumulated experience in each modality. Nevertheless, it is still theoretically informative to ask how performance varies between modalities when learners are presented with similar distributional input (Conway \& Christiansen, 2005; Siegelman $\&$ Frost, 2015). While our tasks were not directly comparable on every aspect, they were matched in the statistical information presented to learners. Despite similar TPs, similar statistical properties and similar procedures in both tasks, and despite the fact that there were slightly more repetitions in the auditory task, children showed higher accuracy in the visual modality throughout development. This result differs from Conway and Christiansen (2005), who found an auditory advantage, but is consistent with Siegelman and Frost (2015), who also found a visual advantage.

One possible reason for this discrepancy is the different presentation rates used in both tasks, which have been found to affect performance differently in the visual and auditory domains: adults show better learning in the visual domain with slower presentation rates and better learning in the auditory domain at faster presentation rates (Emberson et al., 2011). Children also show improved learning of visual triplets with slower presentation rates (Arciuli \& Simpson, 2011). The fact that our presentation rates were not constant across tasks may have contributed to the difference between the modalities. However, it is unlikely that this factor is what underlies the advantage seen in the visual domain for two reasons. First, auditory learning was worse despite being presented at the short and "optimal" presentation rate 
(Emberson et al., 2011). Second, both of our tasks required learning temporal regularities (as opposed to spatial ones), which are supposed to be harder to learn in the visual domain (Emberson et al., 2011; but see Tummeltshammer, Amso, French, \& Kirkham, 2016, for a more complex pattern of results with infants). In other words, despite presenting the auditory stimuli at an easier presentation rate and despite making the visual relations harder to learn by using temporal regularities, visual SL was still better than auditory SL across ages. It is more likely that the visual advantage was affected by the stimuli itself. We used syllables as our auditory stimuli, which are also used in adult studies of auditory SL. However, we used aliens as our visual stimuli, which are more distinguishable compared to the complex, unidentifiable shapes often used in adult studies (Turk-Brown et al., 2005). Adults may find the complex visual shapes used in Turk-Brown et al. harder to process compared to syllables, resulting in worse performance in the visual domain. Since the visual items used here were perhaps easier to process, this helped to match the familiarity of syllables compared to novel visual items. This result suggests that $S L$ is sensitive not only to modality, but also to specific stimulus features: which modality shows better performance is related (among other things) to the familiarity and ease of encoding of the stimuli used in each domain.

While our main goal was to examine the effect of age on SL, our results have important implications for the debate on the domaingenerality of SL. Several studies have uncovered modality-based differences in adults' performance (Conway \& Christiansen, 2005; Siegelman \& Frost, 2015), and our study strengthens and expands on these findings by showing that modality-based differences in SL are present in childhood as well, with different modalities showing different learning rates, different biases and different developmental trajectories. Our findings therefore support modality-sensitive models of SL, which argue that it is not a unitary, a-modal capacity but rather one that is affected by the specific constraints and characteristics of different sensory inputs. Importantly, modality-based effects do not necessarily mean modality-specificity in the sense of a separate and distinct learning mechanism for each domain: SL can show modality effects yet still be, to a degree, domain-general (Emberson et al., 2011; Frost et al., 2015; Saffran \& Thiessen, 2007). For instance, a single learning mechanism could receive information from multiple domains, so the input is first processed and encoded according to its perceptual modality and only then fed into a multi-modal mechanism for further computation and learning. In such a model, modality-based differences arise when the input is encoded. Recent imaging findings provide support for this model by showing that both shared and distinct neural networks are activated while learning in different modalities (Frost et al., 2015). Taken together, our findings add to the growing literature showing that $\mathrm{SL}$ is a modality-sensitive and stimuli-sensitive capacity and predicts that learning outcomes will be affected by the differences between perceptual modalities.

\section{2 | Conclusions}

This study examined the developmental trajectory of visual and auditory SL. The results show that the development of statistical learning is affected by modality: while auditory SL seems age-invariant across childhood, visual SL improves significantly with age. This result reconciles previous studies which showed different patterns in the visual and auditory domain (e.g., Arciuli \& Simpson, 2011; Saffran et al., 1997). These findings also support modality-based differences in SL (Frost et al., 2015), and suggest that SL is not a unitary, stable capacity, but rather one that is sensitive to both modality and stimulus features. Our results highlight the need to develop more implicit SL tasks that can be used from infancy to adulthood, to see if auditory SL is truly invariant from birth or whether it undergoes changes during infancy.

\section{ACKNOWLEDGEMENTS}

We wish to thank Noam Siegelman for comments and help with the statistical analyses; Ram Frost for comments and helpful discussions; and the audience at the BCBL statistical learning workshop. We also want to thank the research assistants at the Living Lab in the Bloomfield Science museum (Tamar Johnson, Maya Enisman, Amira Laufer; Ayelet Gertsovski; Ruth Goldberg), the museum staff; and the children and parents who participated in the studies. The research was funded by an Israeli Science Foundation grant to the second author (grant number 584/16).

\section{ENDNOTE}

${ }^{1}$ This coding system compares the mean of the dependent variable for a given level to the overall mean of the variable. In this case, we compare success on word-first trials to the general mean on all trials. Coding in this way allows us to interpret the intercept of the model as the mean probability for success in a given trial in inverse logit space. If converted to probabilities, the intercept will closely correspond to the mean accuracy score in the task on a scale of 0 to 1 ( 0.55 for $55 \%$ ). Therefore, significant learning in the task is also demonstrated by a significant positive intercept in the model.

\section{REFERENCES}

Arciuli, J., \& Simpson, I.C. (2011). Statistical learning in typically developing children: The role of age and speed of stimulus presentation. Developmental Science, 14, 464-473.

Arciuli, J., \& Simpson, I.C. (2012). Statistical learning is related to reading ability in children and adults. Cognitive Science, 36, 286-304.

Arciuli, J., \& von Koss Torkildsen, J. (2012). Advancing our understanding of the link between statistical learning and language acquisition: The need for longitudinal data. Frontiers in Psychology, 3, 324.

Barr, D.J., Levy, R., Scheepers, C., \& Tily, H.J. (2013). Random effects structure for confirmatory hypothesis testing: Keep it maximal. Journal of Memory and Language, 68, 255-278.

Bertels, J., Boursain, E., Destrebecqz, A., \& Gaillard, V. (2015). Visual statistical learning in children and young adults: How implicit? Frontiers in Psychology, 5, 1541.

Birdsong, D. (Ed.) (1999). Second language acquisition and the critical period hypothesis. New York: Routledge.

Bulf, H., Johnson, S.P., \& Valenza, E. (2011). Visual statistical learning in the newborn infant. Cognition, 121, 127-132.

Cristia, A., Seidl, A., Singh, L., \& Houston, D. (2016). Test-retest reliability in infant speech perception tasks. Infancy, 21, 648-667.

Colombo, J. (2001). The development of visual attention in infancy. Annual Review of Psychology, 52, 337-367. 
Conway, C.M., Bauernschmidt, A., Huang, S.S., \& Pisoni, D.B. (2010). Implicit statistical learning in language processing: Word predictability is the key. Cognition, 114, 356-371.

Conway, C.M., \& Christiansen, M.H. (2005). Modality-constrained statistical learning of tactile, visual, and auditory sequences. Journal of Experimental Psychology: Learning, Memory, and Cognition, 31, 24-39.

Emberson, L.L., Conway, C.M., \& Christiansen, M.H. (2011). Timing is everything: Changes in presentation rate have opposite effects on auditory and visual implicit statistical learning. Quarterly Journal of Experimental Psychology, 64, 1021-1040.

Emberson, L., Misyak, J.B., Schwade, J., Christiansen, M.H., \& Goldstein, M.H. (under review). How abstract is statistical learning? Comparing learning across visual and auditory perceptual modalities in infancy. Presented at the Interdisciplinary Advances in Statistical Learning Conference, Bilbao, Spain.

Ferman, S., \& Karni, A. (2010). No childhood advantage in the acquisition of skill in using an artificial language rule. PLoS One, 5, e13648.

Finn, A.S., \& Hudson-Kam, C.L. (2008). The curse of knowledge: First language knowledge impairs adult learners' use of novel statistics for word segmentation. Cognition, 108, 477-499.

Folia, V., Uddén, J., De Vries, M., Forkstam, C., \& Petersson, K.M. (2010). Artificial language learning in adults and children. Language Learning, 60(s2), 188-220.

Franco, A., Eberlen, J., Destrebecqz, A., Cleeremans, A., \& Bertels, J. (2015) Rapid serial auditory presentation: A new measure of statistical learning in speech segmentation. Experimental Psychology, 62, 346-351.

Frost, R., Armstrong, B.C., Siegelman, N., \& Christiansen, M.H. (2015). Domain generality versus modality specificity: The paradox of statistical learning. Trends in Cognitive Sciences, 19, 117-125.

García-Pérez, M.A., \& Alcalá-Quintana, R. (2010). The difference model with guessing explains interval bias in two-alternative forced-choice detection procedures. Journal of Sensory Studies, 25, 876-898.

García-Pérez, M.A., \& Alcalá-Quintana, R. (2011). Interval bias in 2AFC detection tasks: Sorting out the artifacts. Attention, Perception, and Psychophysics, 73, 2332-2352.

Gathercole, S.E., Pickering, S.J., Ambridge, B., \& Wearing, H. (2004). The structure of working memory from 4 to 15 years of age. Developmental Psychology, 40, 177-190.

Gebhart, A.L., Newport, E.L., \& Aslin, R.N. (2009). Statistical learning of adjacent and nonadjacent dependencies among nonlinguistic sounds. Psychonomic Bulletin and Review, 16, 486-490.

Gervain, J., Nespor, M., Mazuka, R., Horie, R., \& Mehler, J. (2008) Bootstrapping word order in prelexical infants: A Japanese-Italian cross-linguistic study. Cognitive Psychology, 57, 56-74.

Gervain, J., \& Werker, J.F. (2008). How infant speech perception contributes to language acquisition. Language and Linguistics Compass, 2, 1149-1170.

Gómez, R.L., \& Gerken, L. (1999). Artificial grammar learning by 1-year-olds leads to specific and abstract knowledge. Cognition, 70, 109-135.

Janacsek, K., Fiser, J., \& Nemeth, D. (2012). The best time to acquire new skills: Age-related differences in implicit sequence learning across the human lifespan. Developmental Science, 15, 496-505.

Karuza, E.A., Farmer, T.A., Fine, A.B., Smith, F.X., \& Jaeger, T.F. (2014). Online measures of prediction in a self-paced statistical learning task. In Proceedings of the 36th annual meeting of the Cognitive Science Society, Quebec City, Canada.

Kidd, E. (2012). Implicit statistical learning is directly associated with the acquisition of syntax. Developmental Psychology, 48, 171-184.

Kirkham, N.Z., Slemmer, J.A., \& Johnson, S.P. (2002). Visual statistical learning in infancy: Evidence for a domain general learning mechanism. Cognition, 83, B35-B42.

Krogh, L., Vlach, H.A., \& Johnson, S.P. (2012). Statistical learning across development: Flexible yet constrained. Frontiers in Psychology, 3, 598.

Kuhl, P.K. (2004). Early language acquisition: Cracking the speech code. Nature Reviews Neuroscience, 5, 831-843.
Lukács, Á., \& Kemény, F. (2014). Development of different forms of skill learning throughout the lifespan. Cognitive Science, 39, 383-404.

Maye, J., Werker, J.F., \& Gerken, L. (2002). Infant sensitivity to distributional information can affect phonetic discrimination. Cognition, 82, 101-111.

Meulemans, T., Van der Linden, M., \& Perruchet, P. (1998). Implicit sequence learning in children. Journal of Experimental Child Psychology, 69, 199-221.

Misyak, J.B., \& Christiansen, M.H. (2012). Statistical learning and language: An individual differences study. Language Learning, 62, 302-331.

Misyak, J.B., Goldstein, M.H., \& Christiansen, M.H. (2012). Statisticalsequential learning in development. In P. Rebuschat \& J.N. Williams (Eds.), Statistical learning and language acquisition (pp. 13-54). De Gruyter.

Perruchet, P., \& Pacton, S. (2006). Implicit learning and statistical learning: One phenomenon, two approaches. Trends in Cognitive Sciences, 10, 233-238.

Reber, A.S. (1993). Implicit learning and tacit knowledge: An essay on the cognitive unconscious. Oxford: Oxford University Press.

Romberg, A.R., \& Saffran, J.R. (2010). Statistical learning and language acquisition. Wiley Interdisciplinary Reviews: Cognitive Science, 1 , 906-914.

Saffran, J.R. (2001). The use of predictive dependencies in language learning. Journal of Memory and Language, 44, 493-515.

Saffran, J.R. (2002). Constraints on statistical language learning. Journal of Memory and Language, 47, 172-196.

Saffran, J.R. (2003). Statistical language learning: Mechanisms and constraints. Current Directions in Psychological Science, 12, 110-114.

Saffran, J.R., Aslin, R.N., \& Newport, E.L. (1996). Statistical learning by 8-month-old infants. Science, 274, 1926-1928.

Saffran, J.R., Newport, E.L., Aslin, R.N., Tunick, R.A., \& Barrueco, S. (1997). Incidental language learning: Listening (and learning) out of the comer of your ear. Psychological Science, 8, 101-105.

Saffran, J.R., Pollak, S.D., Seibel, R.L., \& Shkolnik, A. (2007). Dog is a dog is a dog: Infant rule learning is not specific to language. Cognition, 105 , 669-680.

Saffran, J.R., \& Thiessen, E.D. (2007). Domain general learning capacities. In E. Hoff \& M. Shatz (Eds.), Blackwell handbook of language development (pp. 68-86). Oxford: Blackwell.

Scaife, M., \& Bruner, J.S. (1975). The capacity for joint visual attention in the infant. Nature, 253, 265-266.

Schlichting, M.L., Guarino, K.F., Schapiro, A.C., Turk-Browne, N.B., \& Preston, A.R. (2017). Hippocampal structure predicts statistical learning and associative inference abilities during development. Journal of Cognitive Neuroscience, 29, 37-51.

Shafto, C.L., Conway, C.M., Field, S.L., \& Houston, D.M. (2012). Visual sequence learning in infancy: Domain-general and domain-specific associations with language. Infancy, 17, 247-271.

Siegelman, N., \& Frost, R. (2015). Statistical learning as an individual ability: Theoretical perspectives and empirical evidence. Journal of Memory and Language, 81, 105-120.

Teinonen, T., Fellman, V., Näätänen, R., Alku, P., \& Huotilainen, M. (2009). Statistical language learning in neonates revealed by event-related brain potentials. BMC Neuroscience, 10, 21.

Thiessen, E.D., \& Erickson, L.C. (2013). Beyond word segmentation A twoprocess account of statistical learning. Current Directions in Psychological Science, 22, 239-243.

Thiessen, E.D., \& Erickson, L.C. (2015). Perceptual development and statistical learning. In B. MacWhinney \& W. O'Grady (Eds.), The handbook of language emergence (pp. 396-414). New York: Wiley-Blackwell.

Thiessen, E.D., Girard, S., \& Erickson, L.C. (2016). Statistical learning and the critical period: How a continuous learning mechanism can give rise to discontinuous learning. Wiley Interdisciplinary Reviews: Cognitive Science, 7, 276-288. 
Thiessen, E.D., \& Saffran, J.R. (2003). When cues collide: Use of stress and statistical cues to word boundaries by 7- to 9-month-old infants. Developmental Psychology, 39, 706-716.

Tummeltshammer, K., Amso, D., French, R.M., \& Kirkham, N.Z. (2016). Across space and time: Infants learn from backward and forward visual statistics. Developmental Science, https://doi.org/10.1111/desc.12474

Turk-Browne, N.B., Jungé, J.A., \& Scholl, B.J. (2005). The automaticity of visual statistical learning. Journal of Experimental Psychology: General, 134, 552-564.

Vaidya, C.J., Huger, M., Howard, D.V., \& Howard Jr., J.H. (2007). Developmental differences in implicit learning of spatial context. Neuropsychology, 21, 497-506.

Vuontela, V., Steenari, M.R., Carlson, S., Koivisto, J., Fjällberg, M., \& Aronen, E.T. (2003). Audiospatial and visuospatial working memory in 6-13 year old school children. Learning and Memory, 10, 74-81.
Yeshurun, Y., Carrasco, M., \& Maloney, L.T. (2008). Bias and sensitivity in two-interval forced choice procedures: Tests of the difference model. Vision Research, 48, 1837-1851.

How to cite this article: Raviv L, Arnon I. The developmental trajectory of children's auditory and visual statistical learning abilities: modality-based differences in the effect of age. Dev Sci. 2018;21:e12593. https://doi.org/10.1111/desc.12593 